\title{
NET production complements endothelial damage
}

NETs might act
as a scaffold
during the
activation of
complement
proteins

The production of neutrophil extracellular traps (NETs) and subsequent endothelial dysfunction have previously been implicated in anti-neutrophil cytoplasmic antibody (ANCA)-associated vasculitis (AAV), but the mechanisms involved in these processes have been unclear. New research has now revealed clear links between ANCA-induced neutrophil activation, NET formation, the alternative complement pathway and endothelial cell damage in murine models of AAV and in patients with AAV who have necrotizing and crescentic glomerulonephritis (NCGN).

NETs are produced by neutrophils as a form of controlled cell death. Necroptosis is the best-characterized form of regulated necrosis and is dependent on receptor-interacting

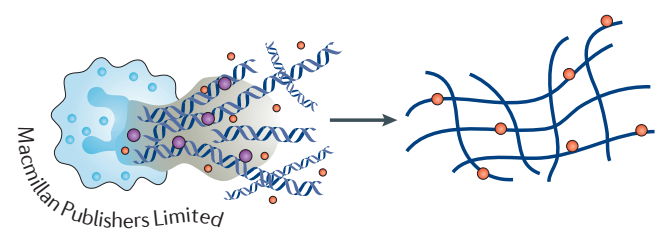

serine/threonine protein kinase 1 (RIPK1) and RIPK3. Blocking RIPK1 in human neutrophils in vitro greatly reduced NET production upon stimulation with ANCAs, a result that was mirrored in neutrophils from mice deficient for RIPK3.

Having established that necroptosis is the pathway necessary for ANCA-stimulated NET production, the researchers proceeded to identify how NETs contribute to the endothelial cell damage that is typically seen in AAV. NETs isolated from ANCAstimulated neutrophils were able to damage endothelial monolayers in vitro: this effect was prevented by either inhibition of necroptosis or degradation of NETs. Curiously, the researchers also noted that NETs produced in response to ANCAs contained complement proteins C5a and C3d. The alternative complement pathway is thought to be involved in endothelial cell damage, and an oral C5a inhibitor is currently under investigation for use in patients with AAV. The authors suggest that NETs might act as a scaffold during the activation of complement proteins.
In a passive transfer mouse model of AAV, treatment with DNase I to degrade NETs prevented the mice from developing disease, and in RIPK3-deficient mice, the same passive transfer model of disease was unable to produce the typical NCGN pathology. Examination of kidney tissue from patients with AAV and NCGN revealed specific staining for phoshphorylated mixed lineage kinase domain-like protein (a protein crucial for necroptosis) in glomerular neutrophils, suggesting that the necroptosis pathway is active in neutrophils in sites of active disease.

"Specific inhibitors of the necroptosis pathway are currently under development or are already being tested in phase II studies," explains corresponding author Adrian Schreiber. "Therefore, necroptosis inhibition could be a potential novel treatment approach for AAV."

Joanna Collison

ORIGINAL ARTICLE Schreiber, A. et al. Necroptosis controls NET generation and mediates complement activation, endothelial damage, and autoimmune vasculitis. Proc. Natl Acad. Sci. USA http://dx.doi.org/10.1073/pnas.1708247114 (2017) 